\title{
Low serum adiponectin level is predictor of arterial stiffness in new onset diabetes after renal transplantation
}

\author{
Kenji Nishimura*, Toshiaki Hirai, Shigeaki Nakazawa, Kazuaki Yamanaka, \\ Hidefumi Kishikawa, Yasuji Ichikawa \\ Department of Urology, Hyogo Prefectural Nishinomiya Hospital, Nishinomiya, Japan; \\ *Corresponding Author: nkennishi3753@nifty.com
}

Received 9 March 2012; revised 8 April 2012; accepted 11 May 2012

\section{ABSTRACT}

Background: The purpose of this study was to determine whether the level of adiponectin in serum of renal transplant patients is associated with arterial stiffness related to new onset diabetes after transplantation (NODAT). Methods: A total of 90 previously non-diabetic patients who underwent renal transplantation between 1999 and 2009 were enrolled. We evaluated their diabetic risk factors, lipid profiles, brachial-ankle pulse wave velocity (baPWV), ankle-brachial blood pressure index (ABPI) and serum adiponectin level before and after transplantation. Results: Eleven $(12.2 \%)$ patients were diagnosed with NODAT and 79 (87.8\%) without (non-NO-DAT). The mean post-transplant serum adiponectin level in NODAT patients was significantly lower than that in non-NODAT patients (11.9 vs. 16.4 $\mu \mathrm{g} / \mathrm{ml}, \mathrm{p}=0.01$ ), whereas the mean baPWV level in NODAT patients was significantly higher (1686 vs. $1468 \mathrm{~cm} / \mathrm{s}, p=0.016$ ). We found a significant inverse correlation between mean pre-transplant serum adiponectin and baPWV level $(r=-0.27$, $p=0.011)$. Furthermore, patients with lower pretransplant serum adiponectin levels $(<20 \mu \mathrm{g} / \mathrm{ml})$ developed NODAT significantly more frequently than those with higher levels $(p=0.038)$ and the mean baPWV level in the former was also significantly higher $(1520 \mathrm{vs} .1412 \mathrm{~cm} / \mathrm{s}, \mathrm{p}=0.047)$. Conclusion: Our results indicate that low pretransplant serum adiponectin level is associated with arterial stiffness in development of NODAT in patients who undergo renal transplantation.

Keywords: Adiponectin; Arterial Stiffness; NODAT; PWV

\section{INTRODUCTION}

New onset diabetes after transplantation (NODAT) is recognized as a potent risk factor of cardiovascular disease (CVD) in renal transplant recipients [1]. Although atherosclerosis is the most important risk factor for CVD after renal transplantation, NODAT in these patients may accelerate atherosclerosis, thereby contributing to CVD. However, it has not been confirmed whether NODAT following renal transplantation contributes to atherosclerosis [2]

Adiponectin, a recently discovered adipocytokine, has been reported to be inversely associated with the risk for diabetes in a non-diabetic population [3], while it was shown to be an independent predictor of NODAT in renal transplant patients [4]. Moreover, it has attracted great attention because of its anti-atherogenic properties [5]. In recent experiments, our group demonstrated that a decreased level of adiponectin in serum after transplantation is significantly associated with insulin resistance in the development of NODAT. Other studies have shown that evaluation of arterial stiffness using brachial-ankle pulse wave velocity (baPWV) might be a useful screening tool to detect CVD in renal transplant recipients $[6,7]$. In the present study, we investigated whether serum adiponectin levels in renal transplant patients are associated with arterial stiffness related to NODAT, using baPWV and ankle-brachial blood pressure index (ABPI).

\section{PATIENTS AND METHODS}

\subsection{Patients}

A total of 90 previously non-diabetic patients who underwent renal transplantation between 1999 and 2009 were enrolled from those undergoing follow-up examinations at our institution. The mean age and post-transplant follow-up duration were 46.9 years old and 64.5 months, respectively. Immunosuppressive drugs administered consisted of basiliximab for induction, then prednisone 
and cyclosporine or tacrolimus, with mycophenolatemofetil, azathioprine or mizoribinefor maintenance. Informed consent was obtained and the study protocol was approved by the local ethics committee.

\subsection{Definitions}

NODAT was defined according to Japan Diabetes Society guidelines based on results of an oral glucose tolerance test (OGTT). Post-transplant homeostasis model assessment for insulin resistance (HOMA-IR) [fasting glucose $(\mathrm{mg} / \mathrm{dl}) \times$ fasting insulin $(\mathrm{mU} / \mathrm{ml})$ divided by 405] [8] and insulinogenic index (I-index) [ratio of increment of plasma insulin $(\mathrm{mU} / \mathrm{ml})$ to that of plasma glucose at $30(\mathrm{mg} / \mathrm{dl})]$ values were obtained using OGTTderived indexes.

\subsection{Laboratory Analysis}

Post-transplant blood samples were collected between August 2007 and March 2010 in a fasting state, and analytic pre-transplant control samples ( 1 - 3 months pretransplant) were also obtained. All samples were immediately centrifuged and stored at $-80^{\circ} \mathrm{C}$ until analysis. Serum adiponectin, c-peptide, and high-sensitivity Creactive protein (hsCRP) levels were measured by BML Laboratories.

\section{4. baPWV and ABPI Measurements}

The levels of baPWV and ABPI were determined using a volume-plethysmograph apparatus (Colin Co. Ltd., Kimaki, Japan), which simultaneously recorded PWV, blood pressure, electrocardiogram, and heart sounds. Subjects were in a supine position after 5 minutes of rest when baPWV measurements were performed bilaterally in the arms and ankles. ABPI was calculated based on the ankle systolic pressure divided by arm systolic pressure.

\subsection{Statistical Analysis}

Data are expressed as the mean \pm standard deviation. A Mann-Whitney $U$-test was used to determine the differences between NODAT and non-NODAT patients. Pearson's or Spearman's correlations and linear regression analyses were used to assess associations between baPWV and serum pre-transplant adiponectin levels. $\mathrm{p}<$ 0.05 was considered to indicate statistical significance. The analyses were conducted using the JMP 6 Japanese Edition (SAS Institute Inc., NC, USA) and StatView-J 5.0 (Hulinks, Inc., Tokyo, Japan) statistical packages.

\section{RESULTS}

\subsection{Comparisons of Characteristics between NODAT and Non-NODAT Patients}

Eleven (12.2\%) patients were diagnosed with NODAT and $79(87.8 \%)$ without (non-NODAT). Characteristics of the study population after dividing into the NODAT and non-NODAT groups are shown in Table 1. NODAT patients showed significantly higher age, hepatisis $\mathrm{C}$ virus positive rate, body mass index, hemoglobin A1c, fasting plasma glucose, HOMA-IR, total cholesterol, and low-density lipoprotein cholesterol, while they had a lower I-index.

In all patients, the mean post-transplant serum adiponectin level $(15.9 \mu \mathrm{g} / \mathrm{ml})$ was significantly lower than the pre-transplant level $(20.0 \mu \mathrm{g} / \mathrm{ml})(\mathrm{p}=0.017)$, whilethe mean pre- and post-transplant serum adiponectin levels in NODAT patients were significantly lower than those in non-NODAT patients (16.2 vs. $20.5 \mu \mathrm{g} / \mathrm{ml}$ and 11.9 vs. $16.4 \mu \mathrm{g} / \mathrm{ml}, \mathrm{p}=0.02$ and 0.01 , respectively) (Figure 1). Also, mean baPWV in the NODAT patients was significantly higher (1686 vs. $1468 \mathrm{~cm} / \mathrm{s}, \mathrm{p}=0.016)$ (Figure 2). There was no significant difference between the groups in regard to ABPI.

\subsection{Correlations between Pre-Transplant Serum Adiponectinand baPWV Level}

There was a significant inverse correlation between mean pre-transplant serum adiponectin and baPWV levels $(r=-0.27, p=0.011)$ (Figure 3). Furthermore, patients with pre-transplant serum adiponectin levels lower than the median $(20 \mu \mathrm{g} / \mathrm{ml})$ developed NODAT significantly more frequently than those with higher than median pre-transplant adiponectin levels $(p=0.038)$ and the mean baPWV level in the former was also significantly higher (1520 vs. $1412 \mathrm{~cm} / \mathrm{s}, \mathrm{p}=0.047)$ (Table 2). However, there was no significant difference between the mean baPWV level in patients with post-transplant serum adiponectin levels lower than the median (15.2 $\mu \mathrm{g} / \mathrm{ml})$ and those with higher post-transplant serum adiponectin levels $(1478$ vs. $1460 \mathrm{~cm} / \mathrm{s}, \mathrm{p}=0.77)$. In addition, the mean baPWV level in patients with a decreases in serum adiponectin levels greater than $50 \%$ before and after renal transplantation was not significantly different from that in those with smaller levels of decrease (1459 vs. $1467 \mathrm{~cm} / \mathrm{s}, \mathrm{p}=0.92$ ).

\section{DISCUSSION}

In renal transplant recipients, arterial stiffness, shown by baPWV level and presence of NODAT, is independently associated with increased risk of CVD. Adiponectin, which has anti-atherogenic properties, is reduced in CVD and NODAT patients. However there is no information regarding the relationship between adiponectin and baPWV level in the development of NODAT, and this is the first study to examine the relationship between serum adiponectin and arterial stiffness in renal transplant patients in regard to NODAT. Our results showed that a 
Table 1. Comparisons of characteristics between NODAT and non-NODAT patients.

\begin{tabular}{|c|c|c|c|}
\hline & $\operatorname{NODAT}(\mathrm{n}=11)$ & Non-NODAT $(\mathrm{n}=79)$ & p-value \\
\hline Age (years) & $53.1 \pm 10.3$ & $45.9 \pm 11.3$ & 0.014 \\
\hline Sex (male/female) & $6 / 5$ & $47 / 32$ & 0.75 \\
\hline Post-transplant follow-up duration (months) & $62.3 \pm 48.5$ & $64.8 \pm 33.2$ & 0.64 \\
\hline Duration of dialysis & $66.1 \pm 51.8$ & $83 \pm 82.1$ & 0.76 \\
\hline Living donor (\%) & 72.7 & 64.4 & 0.96 \\
\hline HCV positive $(\%)$ & 27.3 & 2.2 & 0.0008 \\
\hline Calsinurin inhibitor (number of TAC/CyA) & $9 / 2$ & $53 / 26$ & 0.32 \\
\hline $\operatorname{BMI}\left(\mathrm{kg} / \mathrm{m}^{2}\right)$ & $22.5 \pm 2.3$ & $20.9 \pm 2.7$ & 0.039 \\
\hline Systolic blood pressure (mmHg) & $138 \pm 22$ & $129 \pm 18$ & 0.2 \\
\hline Fasting plasma glucose (mg/dl) & $107.4 \pm 10.1$ & $92.8 \pm 7.6$ & $<0.0001$ \\
\hline $\mathrm{HbA} 1 \mathrm{c}(\mathrm{mg} / \mathrm{dl})$ & $5.6 \pm 0.5$ & $5.1 \pm 0.3$ & 0.003 \\
\hline HOMA-IR & $2.71 \pm 1.52$ & $1.36 \pm 0.84$ & $<0.0001$ \\
\hline I-index & $0.55 \pm 0.36$ & $1.7 \pm 4.36$ & 0.0022 \\
\hline Total cholesterol (mg/dl) & $223 \pm 36$ & $202 \pm 33$ & 0.094 \\
\hline Triglyceride (mg/dl) & $150 \pm 81$ & $131 \pm 78$ & 0.4 \\
\hline High-density lipoprotein cholesterol (mg/dl) & $58 \pm 17$ & $63 \pm 18$ & 0.31 \\
\hline Low-density lipoprotein cholesterol (mg/dl) & $135 \pm 26$ & $113 \pm 26$ & 0.013 \\
\hline Creatinine (mg/dl) & $1.23 \pm 0.35$ & $1.51 \pm 0.57$ & 0.1 \\
\hline e-GFR & $45.6 \pm 12.8$ & $41.6 \pm 14.1$ & 0.36 \\
\hline Post-transplant hsCRP & $0.57 \pm 1.2$ & $0.4 \pm 2$ & 0.1 \\
\hline
\end{tabular}

HCV: hepatitis C virus; TAC: tacrolimus; CyA: cyclosporine; BMI: body mass index; HbA1c: hemoglobin A1c; HOMA-IR: homeostasis model assessment for insulin resistance; I-index: insulinogenic index; e-GFR: estimated glomerular filtration rate; hsCRP: high-sensitivity C-reactive protein. Values for the variables are shown as the mean \pm standard deviation.

Table 2. Correlations between pre-transplant serum adiponectin and NODAT, and baPWV level. baPWV: brachial-ankle pulse wave velocity. Values for the variables are shown as the mean \pm standard deviation.

\begin{tabular}{cccc}
\hline Pre-transplant adiponectin & $\geq$ median & $<$ median & p-value \\
\hline NODAT $(\mathrm{n})$ & 2 & 8 & 0.038 \\
Non-NODAT $(\mathrm{n})$ & 42 & 36 & $1412 \pm 238$ \\
baPWV $(\mathrm{cm} / \mathrm{s})$ & $1520 \pm 272$ & 0.047 \\
\hline
\end{tabular}

low pre-transplant serum adiponectin level is a predictor of arterial stiffness and NODAT in renal transplant patients, with a significant inverse correlation found between mean pre-transplant serum adiponectin and baPWV levels.

Previous studies have demonstrated that a low serum adiponectin level is an independent predictor of high baPWV level in essential hypertension [9] and renal transplant [10] patients. Our results support those findings. However, there was no significant difference between the mean baPWV levels in patients with lower and higher post-transplant serum adiponectin levels or between mean
baPWV levels in patients with changes in serum adiponectin level before and those with such changes after renal transplantation. A recent follow-up study reported a decrease in baPWV level during the first year after renal transplantation [11], while another found a significant positive correlation between baPWV level changes and duration of the follow-up period [12]. It is possible that the present patients were not influenced in the early stage of renal transplantation as compared to the later stage (64.5 months). Furthermore, there are other clinical characteristics in renal transplant recipients other than biochemical parameters that influence baPWV level, such as 


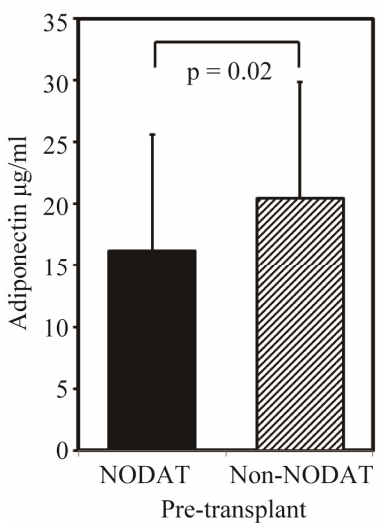

(a)

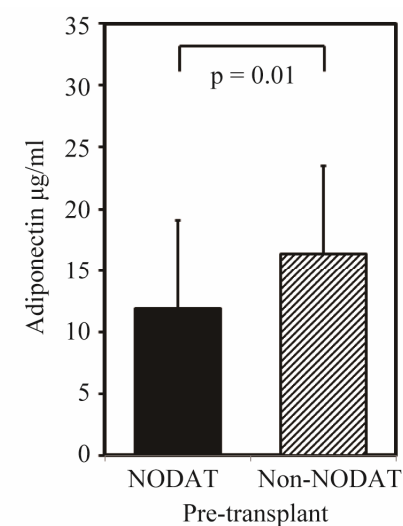

(b)
Figure 1. Comparisons of pre- and post-serum adipinectin levels between NODAT and non-NODAT patients.

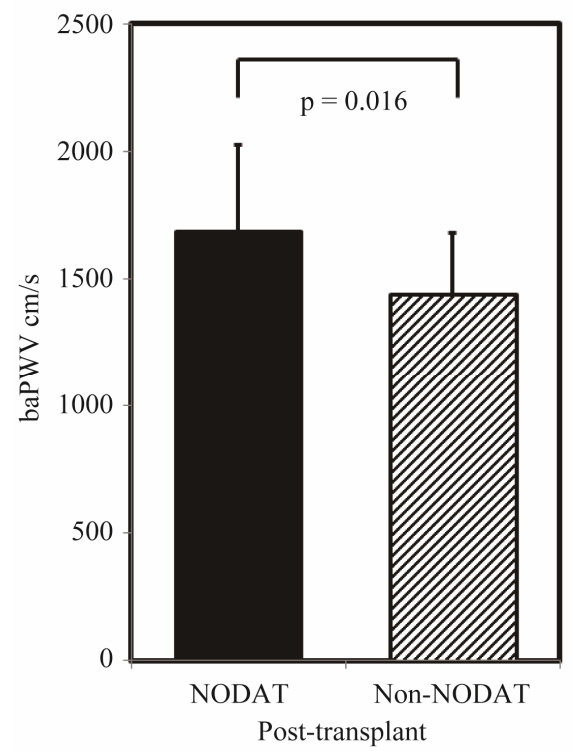

Figure 2. Comparisons of baPWV levels between NODAT and non-NODAT patients. BaPWV: brachial-ankle pulse wave velocity.

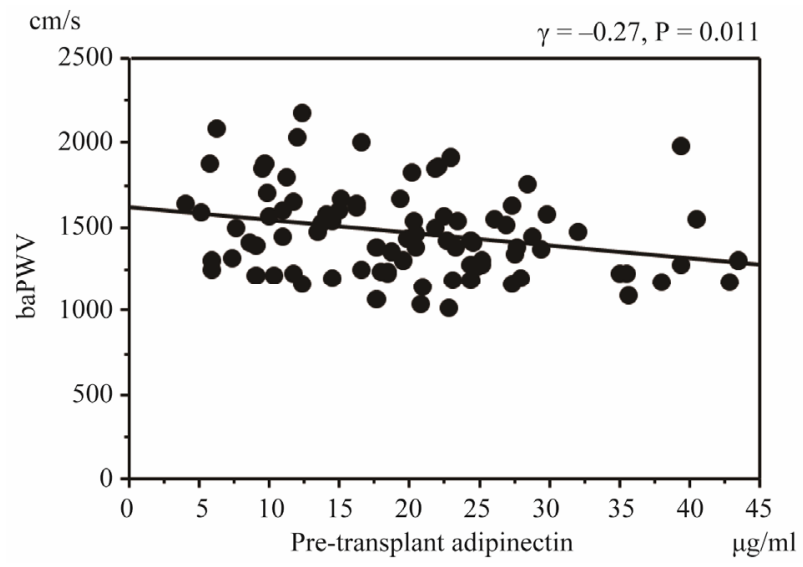

Figure 3. Comparisons between HOMA-IR value and serum adiponectin levels. baPWV: brachial-ankle pulse wave velocity. duration of dialysis, hypertension and diabetes [7]. Thus, effective methods for evaluation of improvement of arterial stiffness after renal transplantation are controversial. Based on our findings, we consider that a low pre-transplant but not post-transplant serum adiponectin level isa very important factor to predict post-transplant baPWV level. Therefore, in patients with a low pre-transplant serum adiponectin level, strict dietary controls or instructions may be important to prevent NODAT and CVD after renal transplantation.

With the increasing number of NODAT patients, it is important to determine which are at high risk for posttransplant CVD and complications. Previous studies of renal transplant populations revealed that recipient and donor age, hypertension, duration of dialysis, diabetes, and acute rejection were related to increased arterial stiffness $[7,13]$. In addition, lower serum magnesium [14] and paraoxonase 1 activity [15] have been reported to be independently associated with arterial stiffness. Our results show a similar association between low pre-transplant serum adiponectin level and arterial stiffness, and that those levels are associated with development of NODAT.

The relationship between adiponectin and baPWV levels is unclear. Several studies have indicated that systemic inflammatory factors accelerate atherosclerosis and increase CVD risk [16,17]. Circulating lipid-related intermediate metabolites may be closely associated with inflammation, oxidative stress, and arterial stiffness in early diabetes [18]. Thus, we speculate that the relationship is due to a reduced anti-inflammatory effect as a consequence of low serum adiponectin level, as also presented in our studies. Adiponectin plays a significant role in anti-inflammatory properties mediated by some inflammatory markers, such as CRP, interleukin-6, tumor necrosis factor-alpha, and others, which are also associated with the development of NODAT [19]. In this study, we measured serum hsCRP in transplant recipients, but found no relationship between NODAT and non-NODAT patients. Therefore, it is possible that other inflammatory markers may influence arterial stiffness in the development of NODAT.

We acknowledge several limitations in the present study. First, there was a small number of subjects enrolled, all of whom were Japanese. Second, our subjects were not chosen in a randomized fashion and the time periods from transplantation to entry varied. Finally, pretransplant baPWV measurements were unavailable. However, the determination of pre-transplant baPWV was beyond the scope of this study, because we did not aim to analyze changes in arterial stiffness before and after renal transplantation. Measurements of baPWV levels at various time points are needed to examine changes in arterial stiffness after renal transplantation, as those differ over time. 


\section{CONCLUSION}

Our results indicate that low pre-transplant serum adiponectin level is apredictor of arterial stiffness and NODAT in renal transplant patients, while we also found a significant inverse correlation between mean pre-transplant serum adiponectin and baPWV levels. Therefore, in patients with low pre-transplant serum adiponectin levels, strict follow-up examinations and prophylactic treatment are needed for prevention of NODAT and CVD after renal transplantation.

\section{REFERENCES}

[1] Pavlakis, M. (2005) New-onset diabetes after transplantation. Current Diabetes Reports, 5, 300-304. doi:10.1007/s11892-005-0027-y

[2] OpazoSaez, A., Kos, M., Witzke, O., Kribben, A. and Nürnberger, J. (2008) Effect of new-onset diabetes mellitus on arterial stiffness in renal transplantation. Transplant International, 21, 930-935. doi:10.1111/j.1432-2277.2008.00702.x

[3] Li, S., Shin, H.J., Ding, E.L. and van Dam, R.M. (2009) Adiponectin levels and risk of type 2 diabetes: A systematic review and meta-analysis. The Journal of the American Medical Association, 302, 179-188. doi:10.1001/jama.2009.976

[4] Bayés, B., Granada, M.L., Pastor, M.C., Lauzurica, R., Salinas, I., Sanmarti, A., Espinal, A., Navarro, M., Bonal, J. and Romero, R. (2007) Obesity, adiponectin and inflammation as predictors of new-onset diabetes mellitus after kidney transplantation. American Journal of Transplantation, 7, 416-422. doi:10.1111/j.1600-6143.2006.01646.x

[5] Wieçek, A., Adamczak, M. and Chudek, J. (2007) Adiponectin-An adipokine with unique metabolic properties. Journal: Nephrol Dial Transplant, 22, 981-988. doi:10.1093/ndt/gfl814

[6] Nishimura, K., Kishikawa, H., Kato, T., Kobayashi, Y., Fujii, N., Takahara, S. and Ichikawa, Y. (2009) Tacrolimus and angiotensin receptor blockers associated with changes in serum adiponectin level in new-onset diabetes after renal transplantation: Single-center cross-sectional analysis. Transplant International, 22, 694-701. doi:10.1111/j.1432-2277.2009.00849.x

[7] Hirai, T., Okuno, A., Souda, T., Yonemoto, S., Yamanaka, K., Fujii, N., Kishikawa, H., Nishimura, K. and Ichikawa Y. (2010) Evaluation of arterial stiffness after successful renal transplantation using brachial-ankle pulse wave velocity. Transplantation Proceedings, 42, 4061-4063. doi:10.1016/j.transproceed.2010.09.083

[8] Matthews, D.R., Hosker, J.P., Rudenski, A.S., Naylor, B.A., Treacher, D.F. and Turner, R.C. (1985) Homeostasis model assessment: Insulin resistance and beta-cell function from fasting plasma glucose and insulin concentrations in man. Diabetologia, 28, 412-419. doi:10.1007/BF00280883

[9] Mahmud, A. and Freely, J. (2005) Adiponectin and arte- rial stiffness. American Journal of Hypertension, 18, 1543 1548. doi:10.1016/j.amjhyper.2005.06.014

[10] Kato, K., Matsuhisa, M., Ichimaru, N., Takahara, S., Kojima, Y., Yamamoto, K., Shiraiwa, T., Kuroda, A., Katakami, N., Sakamoto, K., Matsuoka, T.A., Kaneto, H., Yamasaki, Y. and Hori M. (2008) The impact of new-onset diabetes on arterial stiffness after renal transplantation. Endocrine Journal, 55, 677-683. doi:10.1507/endocrj.K07E-138

[11] Nishioka, T., Akiyama, T., Nose, K. and Koike, H. (2008) Arterial stiffness after successful renal transplantation. Transplantation Proceedings, 40, 2405-2408. doi:10.1016/j.transproceed.2008.07.038

[12] Strozecki, P., Adamowicz, A., Kozlowski, M., Wlodarczyk, Z. and Manitius, J. (2011) Progressive arterial stiffening in kidney transplant recipients. Annals of Transplantation, 30, 30-35.

[13] Ha, C.Y., Kim, J.Y., Paik, J.K., Kim, O.Y., Paik, Y.H., Lee, E.J. and Lee, J.H. (2011) The association of specific metabolites of lipid metabolism with markers of oxidative stress, inflammation and arterial stiffness in men with newly diagnosed type 2 Diabetes. Clinical Endocrinology, 2011. doi:10.1111/j.1365-2265.2011.04244.X

[14] Van Laecke, S., Maréchal, C., Verbeke, F., Peeters, P., Van Biesen, W., Devuyst, O. and Vanholder, R. (2011) The relation between hypomagnesaemia and vascular stiffness in renal transplant recipients. Nephrology Dialysis Transplantation, 26, 2362-2369. doi:10.1093/ndt/gfa728

[15] Gungor, O., Kircelli, F., Demirci, M.S., Tuncel, P., Sisman, A.R., Tatar, E., Hur, E., Asci, G., Ok, E. and Toz, H. (2011) Serum paraoxonase 1 activity predicts arterial stiffness in renal transplant recipients. Journal of Atherosclerosis and Thrombosis, 18, 901-905. doi:10.5551/jat.9175

[16] Hotta, K., Funahashi, T., Arita, Y., Takahashi, M., Matsuda, M., Okamoto, Y., Iwahashi, H., Kuriyama, H., Ouchi, N., Maeda, K., Nishida, M., Kihara, S., Sakai, N., Nakajima, T., Hasegawa, K., Murakami, M., Ohmoto, Y., Nakamura, T., Yamashita, S., Hanafusa, T. and Matsuzawa, Y. (2000) Plasma concentrations of a novel, adipose-specific protein, adiponectin, in type 2 diabetic patients. Arteriosclerosis, Thrombosis, and Vascular Biology, 20, 1595-1599. doi:10.1161/01.ATV.20.6.1595

[17] Provan, S.A., Angel, K., Semb, A.G., Mowinckel, P., Agewall, S., Atar, D. and Kvien, T.K. (2011) Early prediction of increased arterial stiffness in patients with chronic inflammation: A 15-year followup study of 108 patients with rheumatoid arthritis. The Journal of Rheumatology, 38, 606-612. doi:10.3899/jrheum.100689

[18] Kampus, P., Muda, P., Kals, J., Ristimäe, T., Fischer, K., Teesalu, R. and Zilmer, M. (2006) The relationship between inflammation and arterialstiffness in patients with essential hypertension. International Journal of Cardiology, 112, 46-51. doi:10.1016/j.ijcard.2005.08.026

[19] Simpson, K.A. and Singh, M.A. (2008) Effects of exercise on adiponectin: A systematic review. Obesity, 16, 241-256. doi:10.1038/oby.2007.53 